\title{
UTILIZACIÓN DE GERMOPLASMA EN EL MEJORAMIENTO DE LA PAPA
}

\author{
ELSA L. CAMADRO; AMERICO O. MENDIBURU*
}

\begin{abstract}
RESUMEN
Se presentan los fundamentos teóricos de un esquema de mejoramiento genético de la papa que implica: 1) la utilización de germoplasma en el desarrollo de poblaciones tetraploides y diploides con amplia base de variabilidad heredable; 2) el mejoramiento genético de esas poblaciones a través de ciclos de reproducción sexual y selección recurrente; 3) la síntesis de híbridos entre individuos (clones) seleccionados, provenientes de distintas poblaciones, y la evaluación de los mismos sobre la base de su aptitud agronómica.
\end{abstract}

Palabras Claves Adicionales: Haploides, gametos 2n, selección recurrente.

\section{ABSTRACT \\ Utilization of Potato Germolasm for Breeding}

The theoretical rationale of a potato breeding scheme is presented. This scheme involves: 1) utilization of germoplasm in the development of tetraploid and diploid populations with a wide base of inheritable variability; 2) breeding of these populations through cycles of sexual reproduction and recurrent selection; 3) synthesis of hybrids between selected individuals (clones) from different populations and evaluation of these hybrids on the basis of their agronomic aptitude.

Additional index Words: Haploids, 2n gametes, recurrent selection.

El plan de mejoramiento genético óptimo es aquél capaz de capitalizar al máximo las posibilidades que ofrece la especie que se ha de mejorar. La papa común (Solanum tuberosum Grupo Tuberosum) es un tetraploide ( $2 n=4 x=48)$, de herencia tetrasómica, que admite reproducción sexual y asexual, reducción del número cromosómico al nivel diploide $(2 \mathrm{n}=2 \mathrm{x}=24)$ por medio de la haploidización $\mathrm{y}$ restauración del nivel tetraploide por vía asexual (tratamiento con colchicina), sexual (cruzamientos en los que funcionan gametos 2n) o parasexual (fusión de protoplastos).

\footnotetext{
* Ing. Agr., M.S., Ph.D., Facultad de Ciencias Agrarias Universidad Nacional de Mar del Plata; Ing. Agr., M.S., Ph.D., Estación Experimental Agropecuaria Balcarce, Instituto Nacional de Tecnología Agropecuaria. C. C. 276 (7620) Balcarce, Buenos Aires, Argentina.
} 
En los programas convencionales de mejoramiento genético de papa, particularmente en aquellos países que tienen sistemas adecuados de producción de tubérculo-semilla, se utiliza el método de pedigrí, que consiste en la realización de cruzamientos controlados entre progenitores tetraploides seleccionados por rendimiento, resistencia a agentes bióticos y abióticos perjudiciales y otras características agronómicas favorables, seguida de la selección clonal durante varios ciclos. El mejoramiento genético de poblaciones aplicado a poblaciones de germoplasma no adaptado es limitado y está restringido principalmente a los programas de Cornell y Carolina del Norte, en EE. UU., y del Centro Internacional de la Papa, en Perú.

\section{HERENCIA TETRASOMICA}

La herencia tetrasómica presenta ventajas y desventajas para el mejoramiento genético sobre la herencia disómica. De acuerdo con la teoría actualmente aceptada de heterosis en papa (22) (23), las interacciones dentro y entre locus son muy importantes para la determinación del rendimiento. Con un número máximo posible de cuatro alelos diferentes por locus, pueden existir 11 interacciones en un locus tetrasómico. En diploides, por otro lado, el número máximo de alelos diferentes por locus es dos y, consecuentemente, sólo es posible una interacción interalélica. Por ende, el nivel tetrasómico es de mayor potencialidad productiva que el disómico, ya que puede albergar mayor diversidad genética por locus; ello genera la posibilidad de promover respuestas heteróticas no alcanzables en el nivel de ploidía inferior (22). Datos experimentales obtenidos en papa $y$ en otros tetraploides tetrasómicos naturales (alfalfa) e inducidos (maíz y centeno autotetraploides), de acuerdo con lo citado por Bingham (1), sustentan esta teoría.

La herencia tetrasómica, sin embargo, es más compleja que la herencia disómica. Por ello, para realizar cualquier estudio genético se requieren progenies numerosas. Por ejemplo, si se considera un locus con dos alelos, A y a, en un diploide son posibles tres genotipos: $\mathrm{AA}, \mathrm{Aa} \mathrm{y}$ aa. En contraste, en un tetraploide son posibles cinco genotipos: AAAA (cuadruplexo), AAAa (triplexo), AAaa (duplexo), Aaaa (simplexo) y aaaa (nuliplexo). En consecuencia, la segregación en diploides puede ocurrir como resultado del apareamiento de un solo genotipo, Aa, mientras que en tetraploides puede ocurrir por el apareamiento de tres genotipos, AAAa, AAaa, Aaaa. Cuando se autofecunda un individuo heterocigota para un locus, la probabilidad de obtener descendientes homocigotas recesivos es de 1/4 en diploides y de 1/36 en tetraploides duplexos. En adición, fenómenos como la dominancia incompleta y la doble reducción pueden aumentar la complejidad de la herencia tetrasómica en comparación con la disómica. Esta complejidad puede ser un verdadero obstáculo cuando se desea recuperar en la descendencia determinados genotipos recombinantes, especialmente para caracteres controlados por poligenes, como rendimiento o resistencia a algunas enfermedades (30) (34). 
Las dificultades expuestas, en conjunción con las limitaciones impuestas por la cantidad de material que un mejorador puede manejar por el método de pedigrí y la estrecha base genética de la papa cultivada, Solanum tuberosum spp. tuberosum hacen que los avances en el mejoramiento genético en el nivel tetraploide sean lentos y, posiblemente, algunos de ellos vayan acompañados del deterioro en otros. Ello explicaría, en parte, por qué algunas variedades europeas (como Bintje y King Edwuard) o americanas (como Russet Burbank) sigan siendo cultivadas, a más de 70 años de haber sido creadas.

\section{HAPLOIDIZACION}

Hougas y colaboradores (9) encontraron una técnica sencilla, cruzamientos $4 \mathrm{x}$ x 2x, para producir haploides ginogenéticos de papa en grandes números. Hermsen y Verdenius (6) aumentaron la aplicabilidad de esa técnica al introducir un nuevo marcador genético en clones inductores de haploidía, que facilitaran el tamizado de las progenies. Recientemente se han afinado técnicas para producir haploides androgenéticos por cultivo in vitro de anteras (13) (14) (24) (36).

En general, el proceso de haploidización permite al investigador beneficiarse con la herencia disómica, ya que los haploides $(2 n=2 x=24)$ derivan de clones tetraploides $(2 n=4 x=48)$ con herencia tetrasómica. La haploidía provee una forma de explorar la variabilidad genética existente en individuos poliploides y permite obtener genotipos gaméticos élite, que pueden usarse en el mejoramiento genético mediante los procedimientos habituales (cruzamientos, retrocruzamientos, selección) (8). Este proceso va acompañado de una marcada pérdida de vigor, fertilidad — los haploides de papa son en su mayoría androestériles (2) (31) —y productividad en comparación con el progenitor tetraploide, y un incremento en el coeficiente de endocría como resultado de la doble reducción (22).

Muchos caracteres de interés económico, por ejemplo la resistancia a agentes bióticos (Phytophthora, Alternaría. Streptomyces), virus $\mathrm{X}$, virus $\mathrm{Y}$, virus del enrollado de la hoja de papa, nemátodos, etc) y abióticos (heladas, calor, sequía) han sido descriptos en especies silvestres de papa (5).

La mayoría de las especies silvestres son diploides y no es posible obtener descendencia directamente en cruzamientos con tetraploides debido a problemas en el desarrollo del endospermo (a menos que las especies diploides produzcan gametos 2n). Ese problema puede resolverse mediante la duplicación del número cromosómico de las especies diploides con colchicina previamente a la realización del cruzamiento $4 \mathrm{x}-2 \mathrm{x}$, pero este método presenta la desventaja de producir híbridos con herencia tetrasómica. Sin embargo, es posible ampliar la base genética de la papa cultivada mediante cruzamientos entre haploides seleccionados y otros Solanum diploides silvestres y cultivados (8) (10) (16). 
Los híbridos entre haploides de Tuberosum y especies silvestres son, en general vigorosos y fértiles (7) (10) (16) (17), ya que las especies aportan no sólo diversidad genética sino también fertilidad masculina. Dado que los haploides contribuyen con características agronómicas favorables en el híbrido, es posible recuperar progenies agronómicamente aceptables en pocos ciclos de selección (10). Las manipulaciones adicionales que se realizan con posterioridad a la síntesis del híbrido haploide-especie se hacen a expensas de una porción de la heterocigosis inicial del mismo y, por ello, no son convenientes (22). Sin embargo, en muchas situaciones prácticas no es posible profundizar la fase analítica de mejoramiento de genomios antes de la síntesis del híbrido diploide, lo que trae como consecuencia la necesidad de afrontar esa pérdida de heterocigosis. Aparentemente, este es un precio que debe ser pagado ya que, con los métodos corrientes, es casi nulo el progreso que se puede lograr al realizar ciclos de selección por tuberización en poblaciones de especies puras, previamente a la síntesis del híbrido .

\section{POLIPLOIDIZACION}

El producto final del mejoramiento genético debe ser tetraploide ya que ese es, aparentemente, el nivel óptimo de ploidía de la papa cultivada (3).

La restauración del nivel tetraploide puede hacerse en forma asexual, mediante la aplicación de colchicina en tejidos somáticos (32) o por regeneración de plantas a partir de callos derivados de tejido foliar en cultivos in vitro (12); en forma sexual, mediante cruzamientos en los que funcionan gametos 2n (20) o en forma parasexual, mediante la fusión de protoplastos (19) (37).

Las consecuencias genéticas de cada uno de los procesos de poliploidización son diferentes, ya que el nivel de variabilidad genética manifestado por la progenie es una función de aquél presente en los padres y del modo de poliploidización (20). Por ejemplo, si se consideran dos individuos heterocigotas para un locus, $A_{1} A_{2}$ y $A_{3} A_{4}$, con un coeficiente de endocría $\left(F_{2 x}\right)$ igual a $O$, capaces de producir gametos $2 \mathrm{n}$ y de hibridarse en forma sexual y parasexual, la duplicación somática de los cromosomas originará tetraploides dialélicos balanceados, $\mathrm{A}_{1} \mathrm{~A}_{1} \mathrm{~A}_{2} \mathrm{~A}_{2} \quad \mathrm{y} \quad \mathrm{A}_{3} \mathrm{~A}_{3} \mathrm{~A}_{4} \mathrm{~A}_{4}$ considerablemente endocriados $\left(F_{4 x}=1 / 3\right)$. Estos tetraploides mantienen inmodificado el contenido genético de sus contrapartes diploides correspondientes. Por ende, conservarán las resistencias por las que éstos habían sido seleccionados $\mathrm{y}$, en rigor, no cabría esperar que su vigor $\mathrm{y}$ rendimiento se hubieran modificado. Parece correcto interpretar, entonces, que la variación en el valor del coeficiente de endocría sólo refleja el hecho de que en el nivel disómico, cuando $\mathrm{F}_{2 \mathrm{x}}=\mathrm{O}$, se ha logrado el potencial máximo de diversidad genética compatible con este grado de ploidía, mientras que $\mathrm{F}_{4 \mathrm{~s}}=\mathrm{l} / 3$, revela un grado de

\footnotetext{
${ }^{*}$ Hanneman — 1979; Clausen — 1985. Comunicación personal.
} 
capacidad ahora vacante en el nivel tetrasómico donde se puede incorporar mayor diversidad genética. Por otro lado, la hibridación parasexual producirá individuos heterocigotas máximos, $\mathrm{A}_{1} \mathrm{~A}_{2} \mathrm{~A}_{3} \mathrm{~A}_{4}$; en este caso, la transmisión de la heterocigosis será perfecta y las resistencias observadas en el nivel diploide serán combinadas en el tetraploide en un solo genotipo. Sin embargo, para poder evaluar en el campo 10.000 genotipos se necesitarían 10.000 fusiones diferentes. Esta tarea, actualmente, es prácticamente imposible, ya que las técnicas de aislamiento de protoplastos, fusión de los mismos y regeneración de plantas ha sido desarrollada sólo para algunos genotipos en particular. La poliploidización sexual, en cambio, originará individuos con varios grados de heterocigosis y variabilidad genética, evitando la endocría en una medida que dependerá del modo de formación de los gametos $2 \mathrm{n}$ y del número de entrecruzamientos por brazo cromosómico (22).

En el presente, la poliploidización sexual es el método más eficiente y de fácil aplicación para restaurar el nivel tetraploide. Mediante el uso de mutantes meióticos que afectan a los procesos de microsporogénesis, megasporogénesis, o a ambos, es posible transmitir genotipos seleccionados en el nivel diploide al nivel tetraploide en forma casi intacta. Por ejemplo, la orientación paralela de los husos en Anafase II, controlada por el mutante ps, es esencialmente un mecanismo de restitución de la primera división meiótica (RPD) a través del cual se incorpora en los gametos aproximadamente $80 \%$ de la heterocigosis presente en los padres (29). Los mutantes que afectan el apareamiento cromosómico suprimiéndolo o restringiéndolo severamente, como $s y_{2}$ (15), $s y_{3}$ (27) y $\mathrm{sy}_{4}(11)$, combinados con husos paralelos permiten transmitir hasta un $100 \%$ del ordenamiento génico original. Estos mutantes meióticos son de herencia simple (25) (33), de modo que es posible incorporarlos en una población diploide que no los posee y aumentar su frecuencia por selección.

La síntesis final de tetraploides puede hacerse a través de cruzamientos $2 x-2 x$ (poliploidización sexual bilateral, PSB) 0 $4 \mathrm{x}-2 \mathrm{x}$ (poliploidización sexual unilateral, PSU). Para promover la obtención de respuestas heteróticas significativas, los híbridos diploides y tetraploides que intervengan en los cruzamientos deben ser de orígenes diversos y el resultado de un intenso trabajo de mejoramiento genético (22). En tetraploides obtenidos por PSB y PSU se han observado respuestas heteróticas en términos de rendimiento de tubérculos (21) (26) (4) (18), vigor del gametofito masculino (36), vigor vegetativo y vigor de plántulas (28). Estas respuestas han ido acompañadas de una gran uniformidad en la progenie, debido a la alta homogeneidad de los gametos formados por RPD. 


\section{MEJORAMIENTO GENÉTICO}

Por todo lo expuesto, y sobre la base de los esquemas analíticosintéticos de mejoramiento genético de la papa desarrollados por Chase (3) y Mendiburu y colaboradores (22), se propone:

1. La utilización de germoplasma en el desarrollo de poblaciones tetraploides y diploides, con amplia base de variabilidad heredable.

2. El mejoramiento genético de esas poblaciones a través de ciclos de reproducción sexual y selección recurrente.

3. La síntesis de híbridos entre individuos (clones) seleccionados provenientes de distintas poblaciones y la evaluación de los mismos sobre la base de su aptitud agronómica.

La elección de los materiales que se han de utilizar dependerá de la disponibilidad de los mismos para el mejorador y de los objetivos de mejoramiento que se deseen alcanzar. Sin embargo, para promover respuestas heteróticas en los híbridos que se sinteticen se debe asegurar un grado óptimo de diversidad genética entre fuentes de germoplasma.

La selección deberá realizarse en forma independiente en cada población, para mantener e incrementar la diversidad genética. Los criterios que se pueden emplear en dicha selección son: 1) en los dos primeros ciclos, habilidad para tuberizar y 2) en los ciclos sucesivos, rendimiento de tubérculos y comportamiento frente a agentes bióticos y abióticos perjudiciales, con la finalidad de incorporar resistencias múltiples en el híbrido final. En las poblaciones diploides se deberá seleccionar, además, por producción de gametos 2n por RPD.

Los ciclos de selección pueden ser del tipo "semilla-semilla" o "semilla-tubérculo-semilla". Los ciclos "semilla-semilla" son más fáciles de llevar a cabo, pero presentan la desventaja de que en ellos sólo se controla el progenitor femenino, sobre el que se cosechan bayas de polinización libre. En los ciclos "semilla-tubérculo-semilla" se realizan cruzamientos controlados entre progenitores seleccionados para obtener la semilla con la que se iniciará el ciclo siguiente. Ello hace que la realización de este tipo de ciclo sea más laboriosa pero, si se dispone de facilidades para realizar los cruzamientos en el mismo año en el que se practica la selección, el progreso que se logra por año es mayor.

A partir del cuarto ciclo de selección recurrente se puede comenzar a realizar cruzamientos controlados entre clones destacados de las diferentes poblaciones, para explorar las posibilidades de obtener respuestas heteróticas dentro y entre niveles de ploidía.

En síntesis, se propone un plan de mejoramiento genético de la papa que implica:

1) El manejo de poblaciones de ploidías distintas (x y 4x), lo cual permitiría comparar — eventualmente- el progreso genético del mejoramiento de caracteres análogos en condiciones de herencia tetrasómica y herencia disómica.

2) Manipulaciones de ploidía: obtención de haploides $(2 n=2 x=: 24)$ a partir de tetrapoides $(2 n=4 x=48)$ identificación $y$ 
uso de gametos $2 \mathrm{n}$ y producción de tetraploides mediante cruzamientos $4 x-2 x$ y $2 x-2 x$.

3) Detección y uso de la heterosis que se produce en la progenie de cruzamientos entre individuos (clones) adaptados a las condiciones del medio local, aunque considerablemente distantes desde el punto de vista genético, por haber sido seleccionados en poblaciones independientes.

\section{REFERENCIAS BIBLIOGRÁFICAS}

1. Bingham, E. T. 1980. Maximizing heterozygosity in autopolyploids. p. 471-489, In: Lewis H. (ed.). Poliploidy, Biological Relevance. New York: Plenum Press.

2. Carrol, C. P. 1975. The inheritance and expression of sterility in hybrids of dihaploid and cultivated diploid potatoes. Genetica 45: 149-162.

3. Chase, S. S. 1963. Analytic breeding in Solanum tuberosum. L. A. sheme utilizing parthenotes and other diploid stocks. Can. J. Genet, Cytol, 5 (4) : 359-363.

4. De Jong, H. Tai, G.C.C. 1977. Analysis of 4x-2x hybrids in cultivated potatoes. Potato Res. 20: 111-121.

5. Hanneman, Jr. R. E.; Bamberg, J. B. 1986. Inventory of tuberbearing Solanum species. University of Wisconsin. College of Agricultural and Life Science, Bull 533.

6. Hermsen, J. G. Th.; Verdenius, J. 1973. Selection from S. tuberosum group Phureja of genotypes combining high frecuency haploid induclion with homozygosity for embryospot. Euphytica 22: 244-259.

7. Hermundstad, S. A.; Peloquin, S. J. 1985. Male fertility and 2n pollen production in haploid-wild species hybrids. Am. Potato J. 62 (9) : 479-488.

8. Hougas, R. W.; Peloquin, S. J. 1958. The potential of potato hapioids in breeding and genetic research, Am. Potato J. 35: 701-707.

9. Hougas, R. W.; Peloquin, S. J.; Ross, R. W. 1958. Hapioids of the common potato. J. Hered. 49: 103-107

10. Iwanaga, M. 1980. Breeding at the $2 x$ level for combined pest and disease resistance using wild species and extracted hapioids form selected tetraploid clones. In: Utilization of the genetic resources of the potato III. Report of the Planning Conference 1980. pp. 110-123. CIP Lima, Peni.

11. Iwanaga, M. 1984. Discovery of a synaptic mutant in potato hapioids and its usefulnes for potato breeding. Theor. Appl. Genet. 68: 87-93. 
12. Jacobsen, E. 1978. Doubling dihaploid potato clones via leaf tissue culture. Z. Pflanzenzucht 80: 80-82.

13. Jacobsen, E.; Sopory, S. K. 1978. The influence and possible recombination of genotypes on the production of microspore embryoids in anther cultures of Solanum tuberosum and dihaploid hybrids. Theor, Appl. Genet. 52: 119-123.

14. Johansson, L. 1986. Improved methods for induction of embryogenesis in anther cultures of Solanum tuberosum. Potato Res.. 29 (2) : 179-190.

15. Johnston, S. A.; Hanneman, R. E. Jr.; Ruhde, R. W. 1981. Identification of an asynaptic variant in Solanum cotnmersonii (Abs.) Am. Potato J. 58 (10) : 506.

16. Leue, E. F.; Peloquin. S. J. 1980. Yield and relative maturity of diploid $\mathrm{F}_{1}$ hybrids of haploids $\mathrm{x}$ wild species. Am. Potato J. 59(10) ; 475-476.

17. Liberal, M. T. 1966 Fertilidade de populações em $F_{1}$ e $F_{2}$ envolvendo haplodies de Solanum tuberosum L., e diversa especies diploides. Peq. Agrop. Bras. 1: 165-172.

18. McHale, N. A.; Lauer, F. I. 1981. Breeding value of 2n pollen from diploid hybrids and Phureja in $4 \mathrm{x}-2 \mathrm{x}$ crosses in potatoes. Am. Potato J. 58: 365-374.

19. Melchers, G. 1978. Potatoes for combined somatic and sexual breeding methods: plants from protoplast and fusion of protoplast of potato and tomato, p. 306-311. In: Alfermann, A. W. Reinhard, E. (eds) . Production of natural compounds by cell culture methods. GSF. München.

20. Mendiburu, A. O., Peloquin, S. J. 1976. Sexual polyploidization and depolyploidization: Same terminology and definitions. Theor, Appl. Genet, 48: 137-143.

21. Mendiburu, A. O.; Peloquin, S. J. 1977. The significance of 2n gametes in potato breeding. Theor. Appl. Genet, 49: 53-61.

22. Mendiburu, A. O. Peloquin, S. J.; Mok, D. W. S. 1974. Potato breeding with hapioids and 2n gametes. p. 249-258. In: Kasha, K. J. (ed.) proceedings of the International Symposiuia on Hapioids in Higher Plants. Guelph, Canadá: University of Guelph.

23. Mendoza, H. A.; Haynes, F. L. 1974. Genetic basis of heterosis for yield in the autotetraploid potato. Theor. Appl. Genet. 45: 21-25.

24. Mix G. 1983. Production of dihaploid plantlets from anthers of autotetraploid genotypes of Solanum tuberosum L. Potato Res. 26: 63-67.

25. Mok, D. W. S.; Peloquin, S. J. 1973. The inheritance of three mechanisms of diplandroid formation. Am. Potato J. 50: 336- 
337.

26. Mok, D. W. S.; Peloquin, S. J. 1975. Breeding value of 2n pollen (diplandrois) in tetraploid $\mathrm{x}$ diploid cresses in potato. Theor. Appl. Genet. 46: 307-314.

27. Okwuagwu, C. O.; Peloquin, S. J. 1981. A method of transferring the intact parental genotype to the offspring via meiotic mutants. Am. Potato J. 58: 512-513 (Abstr.).

28. Parfill, D. E.; Peloquin, S. J. 1977. Variation of vine and tuber yield as a function of harvest date and cultivar. Am. Potato J. 54: 411-417.

29. Peloquin, S. J. 1981. Chromosomal and cytoplasmic manipulations. p. 117-150. In; Frey, K. J. (ed.) . Plant Breeding II. Ames, la,: The lowa State Univer-sity Press.

30. Rieman, R. W.; Hooker, W. J.; Krantz, F. A.; Werner, H. O. 1956. Potato improvement through parental line breeding. Am. Potato J. 33: 31-323.

31. Ross, R. W.; Peloquin, S. J. Hcugas, R. W. 1964. Ferlility in hybrids from $S$. phureja. and S. tuberosum mating. Europ. Potato J. 10: 37-52.

32. Ross, R. W.; Dionne, L. A.; Hougas, R. W. 1967. Doubling of chromosome number of selected Solanum genotypes. Europ. Potato J. 10: 37-52.

33. Saad, M. S. 1983. Synaptic mutant $\left(s y_{3}\right)$ of potato: Genetics; expression within ovules; and univalent behavior. M. S. Thesis. University of Wisconsin. Madison.

34. Simmonds, N. W. 1969. Prospects of potato improvement. Pentlandfield 1837. Scott. Pl. Breed, Sta. 48th. Ann. Rep. 1968-69.

35. Simon, P. W.; Peloquin; S. J. 1976. Pollen vigor as a function of mode of 2n gamete formation in potatoes. J. Hered. 67: 204208.

36. Wenzel, G.; Sopory, S. K. 1978. Production and utilization of dihaploid or Monohaploid potatoes, p. 303-305. In: Alfermann, A. W.; Reinhard, E. (eds.). Production of natural compounds by cell culture methods. GSF München.

37. Wenzel, G.; Schieder, O.; Przewozny, T.; Sopory, S. K.; Melchers, G. 1979. Comparison of single cell culture derived Solanum tuberosum, L. plants and a model for their application in breeding programs. Theor. Appl. Genet. 55: 4455. 